\title{
Article \\ Effect of Different Physical Cross-Linkers on Drug Release from Hydrogel Layers Coated on Magnetically Steerable 3D-Printed Microdevices
}

\author{
Roberto Bernasconi ${ }^{1, *(D)}$, Fabio Pizzetti ${ }^{1}\left(\mathbb{D}\right.$, Arianna Rossetti ${ }^{1}$ (D) Riccardo Perugini $^{1}$, Anna Nova ${ }^{1}$, \\ Marinella Levi ${ }^{2}$ and Filippo Rossi ${ }^{1}$ (D)
}

1 Department of Chemistry, Materials and Chemical Engineering "Giulio Natta”, Politecnico di Milano, Via Mancinelli 7, 20131 Milano, Italy; fabio.pizzetti@polimi.it (F.P.); arianna.rossetti@polimi.it (A.R.); riccardo.perugini@polimi.it (R.P.); anna.nova@mail.polimi.it (A.N.); filippo.rossi@polimi.it (F.R.)

2 Department of Chemistry, Materials and Chemical Engineering "Giulio Natta", Politecnico di Milano, Piazza Leonardo da Vinci 32, 20133 Milano, Italy; marinella.levi@polimi.it

* Correspondence: roberto.bernasconi@polimi.it; Tel.: +39-022-399-3150

\section{check for} updates

Citation: Bernasconi, R.; Pizzetti, F.; Rossetti, A.; Perugini, R.; Nova, A.; Levi, M.; Rossi, F. Effect of Different Physical Cross-Linkers on Drug Release from Hydrogel Layers Coated on Magnetically Steerable 3D-Printed Microdevices. Technologies 2021, 9, 43. https://doi.org/10.3390/ technologies 9020043

Academic Editors: Nam-Trung Nguyen and Dennis Douroumis

Received: 2 May 2021

Accepted: 11 June 2021

Published: 18 June 2021

Publisher's Note: MDPI stays neutral with regard to jurisdictional claims in published maps and institutional affiliations.

Copyright: (c) 2021 by the authors. Licensee MDPI, Basel, Switzerland. This article is an open access article distributed under the terms and conditions of the Creative Commons Attribution (CC BY) license (https:// creativecommons.org/licenses/by/ $4.0 /)$.

\begin{abstract}
In the last few decades, the introduction of microrobotics has drastically changed the way medicine will be approached in the future. The development of untethered steerable microdevices able to operate in vivo inside the human body allows a high localization of the therapeutical action, thus limiting invasiveness and possible medical complications. This approach results are particularly useful in drug delivery, where it is highly beneficial to administer the drug of choice exclusively to the target organ to avoid overdosage and side effects. In this context, drug releasing layers can be loaded on magnetically moveable platforms that can be guided toward the target organ to perform highly targeted release. In the present paper, we evaluate the possible application of alginate hydrogel layers on moveable platforms manufactured by coupling additive manufacturing with wet metallization. Such alginate layers are reticulated using three different physical crosslinkers: $\mathrm{Ca}, \mathrm{Zn}$ or Mn. Their effect on drug release kinetics and on device functionality is evaluated. In the case of alginate reticulated using $\mathrm{Mn}$, the strongly $\mathrm{pH}$ dependent behavior of the resulting hydrogel is evaluated as a possible way to introduce a triggered release functionality on the devices.
\end{abstract}

Keywords: microrobots; 3D printed; drug delivery; hydrogels; alginate

\section{Introduction}

Since ancient times, humankind has discovered that specific substances, both available in nature and synthetic, can be used to cure or even prevent a large number of pathologies. Starting from a primordial knowledge based on herbalism and natural ingredients [1], pharmacology has evolved in modern times into a systematic and technologically advanced science. However, while the research on active principles has significantly progressed, the quest for new administration routes has not proceeded in a comparable way. Even nowadays, the majority of medical preparations follow pharmacokinetics still based on poorly controllable distribution routes in the body. Enteral, intravenous and intramuscular administration all rely on blood-mediated distribution of the drug, while transdermal delivery relies on diffusion through the skin [2]. Indiscriminate transport in the whole body translates into higher doses required to reach a therapeutic concentration in the target organ, with possible dosage-related counterindications [3]. Moreover, non-optimal drug usage may possibly induce drug resistance in the case of pathogens [4] or cancer cells [5].

Starting from these premises, it is evident that targeting drug delivery is a matter of major importance in modern pharmacology [6,7]. In the last few decades, a wealth of smart delivery approaches has been proposed at the laboratory scale: liposomes [8,9], polymeric thin layers [10], DNA nanostructures [11], dendrimers [12], micelles [13] and biocompatible nanoparticles [14-16]. All these approaches partially addressed the problem 
of temporal control over release, but did not significantly address the problem of carrying the drug directly in correspondence of the target organ. One of the most promising approaches to carry out this task is to load the drug-bearing material on remotely guided microrobots able to navigate the body in vivo and reach the target organ [17,18]. Such devices have been developed in the last few years and their potential efficiency for targeted drug administration has been widely demonstrated [19,20]. Biomedical microrobots are, in the vast majority of the cases, actuated by applying controlled, non-invasive magnetic fields [21]. They can be manufactured in a wide dimensional range, from millimeters [22,23] to a few micrometers $[24,25]$, according to the characteristic dimensions of the target organ (e.g., centimeters for the gastrointestinal apparatus or micrometers for some blood vessels).

From the fabrication point of view, magnetically guidable microdevices can be conveniently fabricated using many different techniques. The most interesting, in terms of customizability and flexibility, is probably 3D printing [26]. Drug releasing devices can be printed directly using a drug loadable soft polymer $[25,27,28]$ or using rigid materials that are subsequently coated with drug-releasing polymers [23,29]. In both cases, hydrogels are between the most interesting materials usable [30]. These natural macromolecules are well-known for their biocompatibility, efficiency in drug loading and ease of manufacturing. Furthermore, they can be opportunely functionalized to release drugs only in well-specified conditions (of $\mathrm{pH}$, temperature, etc.) [31]. In this context, we recently demonstrated that untethered microdevices can be fabricated by coupling $3 \mathrm{D}$ printing with wet metallization [22] and that the same technique can be used to realize hydrogel-coated microrobots for controlled drug release [32].

In the present work, we explored the effect of biocompatible crosslinkers alternative to calcium chloride on the drug release properties of alginate layers coated on 3D-printed untethered microdevices. Following the research trend recently started by our group [22,23,33], we employed 3D printing in the form of stereolithography to efficiently manufacture miniaturized moveable platforms that we subsequently coated with different functional metallic layers. In detail, microrobots were coated with CoNiP to allow magnetic actuation and with gold to provide a biocompatible surface. The moveable platforms obtained in this way were then coated with alginate hydrogels crosslinked with either $\mathrm{ZnCl}_{2}$ or $\mathrm{MnCl}_{2}$. These two selected alternative crosslinkers have recently been investigated by Da Silva et al. [34], who did not verify their drug release properties. Consequently, the first aim of the present work is to verify the applicability of alginate layers on shape-optimized moveable platforms and to evidence differences in drug delivery properties. The second main aim of the work is to investigate alternative methodologies to trigger release from alginate exploiting $\mathrm{pH}$ variations. In our previous paper [32], we functionalized alginate hydrogels by introducing a $\mathrm{pH}$ cleavable bond that allowed drug release only in a well-defined $\mathrm{pH}$ range. By doing this, we were able to trigger drug release according to the $\mathrm{pH}$ of the environment crossed by the device. The main drawback of the approach is represented by the necessity to carry out a chemical synthesis, which can potentially damage the molecule loaded and must be specifically designed for each drug. Da Silva et al. evidenced that Mn-reticulated alginate mechanically degraded at low $\mathrm{pH}$. This particularity was employed to implement a $\mathrm{pH}-$ governed instantaneous release functionality on the microdevices. From the applicative point of view, microdevices carrying $\mathrm{pH}$-sensitive hydrogels can find potential application in the gastrointestinal tract, which is characterized by significant $\mathrm{pH}$ variations [35].

\section{Experimental Methods}

\subsection{Microdevices 3D Printing}

The miniaturized devices employed in the present work were 3D-printed and metallized following our previous work [33]. Concisely, the geometry of the devices was initially optimized and their 3D model was designed using Solidworks (Dassault Systèmes, France). The geometry was optimized for stereolithography using Nauta+ (DWS, Thiene, Italy) and the 3D model was sliced with Fictor (DWS, Thiene, Italy). Micro-stereolithography was carried out using a model $028 \mathrm{~J}$ Plus setup (DWS, Thiene, Italy) and the resulting prints 
were post-cured by exposition to UV radiation for $30 \mathrm{~min}$. The $028 \mathrm{~J}$ Plus setup comprises a galvanometer control and a laser able to yield a power of $30 \mathrm{~mW}$ at a wavelength of $405 \mathrm{~nm}$, with a beam spot diameter of $22 \mu \mathrm{m}$.

\subsection{Microdevices Metallization}

A detailed description of the metallization process can be retrieved in our previous work [33]. Briefly, the printed devices were removed from printing supports and coated with a first layer of copper to make their surface conductive. Subsequently, they were coated with CoNiP exploiting a barrel-like metallization approach. At the end of the CoNiP metallization step, the procedure was varied with respect to the cited literature reference. In place of $\mathrm{Cu}$ and $\mathrm{Ag} / \mathrm{TiO}_{2}$, the samples were coated (always via barrel-like plating) with a gold layer from electrolytic deposition in a cyanide-free electrolyte (SG-Au 340 bath from SG Galvanobedarf $\mathrm{GmbH}$ ). The following parameters were employed: $5 \mathrm{~mA} / \mathrm{cm}^{2}$, moderate stirring, $50^{\circ} \mathrm{C}$. At the end of the gold plating step, the samples were thoroughly washed with deionized water and dried with nitrogen.

\subsection{Hydrogel Application}

To apply the hydrogel layers on the devices, the latter were dipped sequentially into the polymer aqueous solutions and then in the physical crosslinker in order to deposit the layers (Figure S7). With the goal of obtaining a homogeneous coating of the device, a custom-made structure (Figure S8) was utilized. The device was suspended to a very thin nylon wire, preventing it from touching any part of the beakers containing the solutions. Initially, the device was immersed into a $2 \% \mathrm{~m} / v$ pure sodium alginate solution (step a in Figure S7). This step was performed inside an ultrasonic bath in order to favor the penetration of the alginate in the scaffold. Then, the device was removed from the alginate solution and immersed into a second solution containing the physical cross-linker (step $b$ in Figure S7). Following the work by Da Silva et al. [34], three physical crosslinkers were employed: $\mathrm{MnCl}_{2}\left(1 \% w / v\right.$ in DI), $\mathrm{ZnCl}_{2}\left(1 \% w / v\right.$ in DI) and $\mathrm{CaCl}_{2}(1 \% w / v$ in DI).

\subsection{Microdevices Characterization}

SEM was performed by means of a Zeiss EVO 50 setup, suitably equipped with an Oxford Instruments Model 7060 EDS module. The magnetic properties of the devices were determined using of a Princeton Measurement Corp. MicroMag 3900 vibrating sample magnetometer (VSM). The roughness of the deposited gold layer was evaluated using a UBM Microfocus laser profilometer.

\subsection{In Vitro Drug Delivery}

Drug release was investigated in simulated physiological conditions: at $37^{\circ} \mathrm{C}$ and $5 \% \mathrm{CO}_{2}$, in a phosphate-buffered saline solution (PBS, pH 7.4). In detail, each device was placed in excess of PBS $(2.5 \mathrm{~mL})$ and aliquots were collected at defined time points, replacing them with an equal volume of fresh solution in order to preserve the diffusion regime between the device and the release environment. Percentages of released Rhodamine B (RhB) were then measured by UV spectroscopy at $570 \mathrm{~nm}$.

\subsection{Magnetic Actuation}

Devices were remotely actuated employing the Octomag magnetic manipulation setup [36]. Microrobots were permanently magnetized along the direction perpendicular to their axis by placing them on a strong $\mathrm{NdFeB}$ magnet. Then, a rotating magnetic field characterized by varying intensity and frequency $(\tau)$ was applied thanks to the Octomag. Uncoated and hydrogel-coated devices were actuated inside a water-filled glass basin to avoid hydrogel desiccation. Their motion was tracked and their speed determined using the software Tracker. 


\section{Results and Discussion}

\subsection{Shape Optimization and 3D Printing}

Recently, we described the use of magnetically moveable scaffold-like architectures to support and transport $\mathrm{pH}$-sensitive hydrogels [32]. The choice of using a scaffold-like design was motivated by the capability of a porous structure to host a good quantity of hydrogel inside its pores. Retrospectively, however, more optimized shapes were evaluated to increase the quantity of hydrogel loaded. The inspiration for a more suitable design was taken from the so-called honey dipper (Figure S1), a common household device normally employed to dose highly viscous fluids (e.g., honey). The particular shape of this tool, characterized by the presence of different parallel plates, is optimized to efficiently retain the fluid in which it is immersed. Since the alginate solution is a viscous fluid prior to gelation, the same concept can be transferred to the design of untethered microdevices. Millimetric honey dippers, characterized by the dimensions detailed in Figure S2, were designed and printed using stereolithography. In analogy with our previous work [32], dimensional features were kept in the few $\mathrm{mm}$ to hundreds $\mu \mathrm{m}$ dimensional range. This is a consequence of the potential use of the devices in the gastrointestinal apparatus, whose tracts present characteristic dimensions compatible with these microdevices. A typical batch of devices was composed of 10 to 15 units and it was successfully printed in a roughly 30 min timespan. Devices were printed exploiting small supports connected to individual printing bases (Figure 1a). At the end of the 3D printing step, devices were removed from the supports using a cutter (Figure 1b).
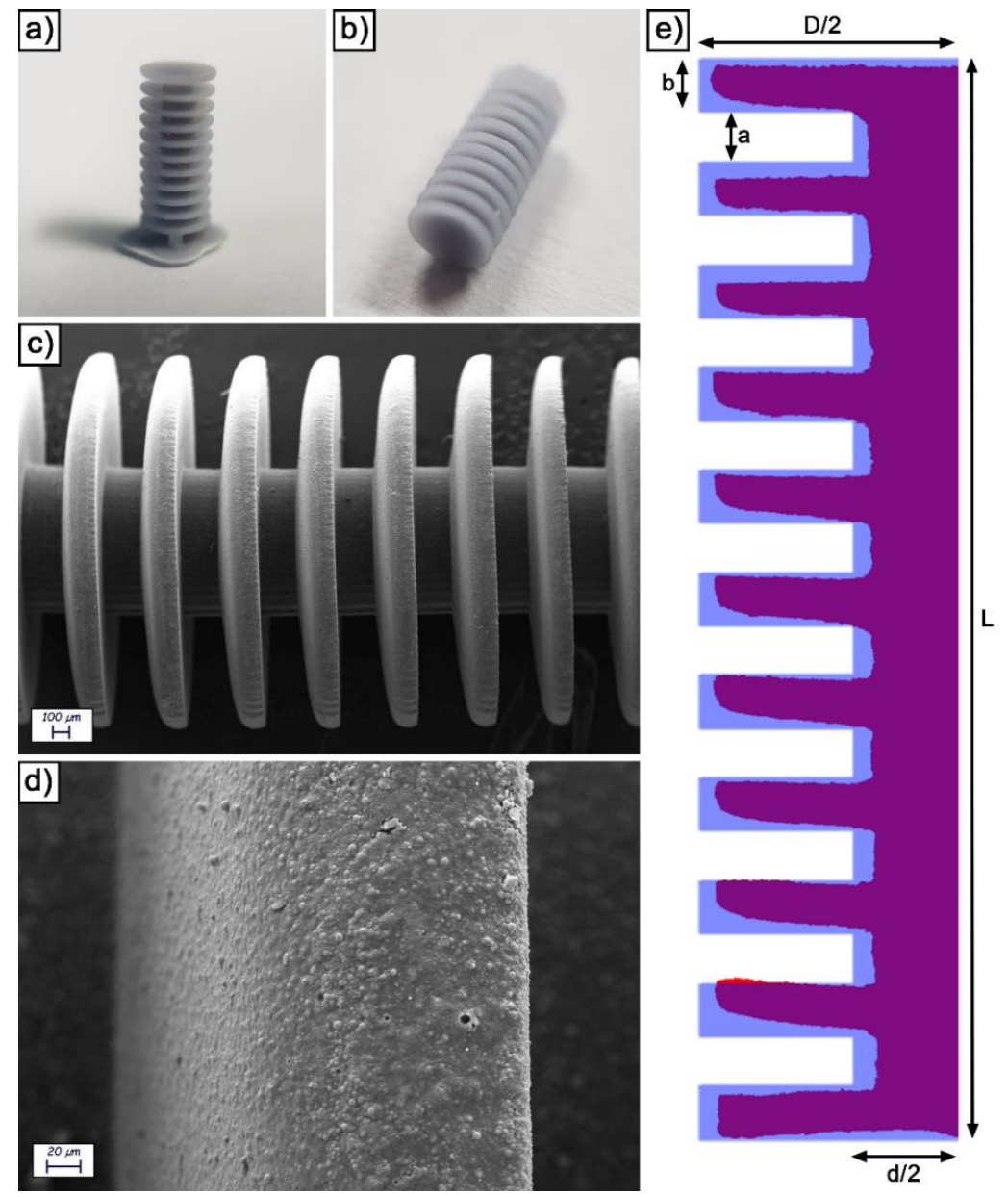

Figure 1. Visual appearance of an as printed device before (a) and after (b) printing supports removal; SEM images of an as printed device observed at $100 \mathrm{X}(\mathbf{c})$ and $1000 \mathrm{X}(\mathrm{d})$; visual comparison between the experimental and theoretical section of a device (e). 
As-printed devices were characterized from the morphological and dimensional point of view. Figure 1c is a low-magnification SEM image, in which the general structure of the devices is clearly visible. As expectable from a micrometric SLA printing, the edges are considerably rounded due to the comparatively large laser beam size $(22 \mu \mathrm{m})$. By increasing the magnification (Figure 1d), the microstructure of the SLA resin is evident. In particular, it presents a relatively rough surface due to the presence of a silica/aluminate filler inside. The latter is clearly visible in the form of spherical particles by further increasing the magnification (Figure S3).

To evaluate dimensional conformity to the original 3D model, devices were sectioned and inglobated inside an epoxy resin. The surface was then polished, yielding the result visible in Figure S4. The profile of the section was then extracted and superimposed to the theoretical one (Figure 1e). In general, the experimental profile is relatively adherent to the 3D model. The comparison, however, confirms the edge rounding observed at the SEM and allows to quantify the deviation from the ideality (Table S1). Large features present experimental dimensions relatively adherent to the corresponding theoretical values ( $98.9 \%$ for $L, 95.8 \%$ for $D$ and $84.6 \%$ for $\mathrm{d}$ ). The largest deviations from the ideality were observed on the stacked disks that constitute the body of the device, which are also the smallest features of the design and present the most challenging printing conditions. In detail, the mean thickness of the disks (b) is only $61.3 \%$ of the theoretical value, while the distance between them (a) is $140.5 \%$ of the theoretical value. Both these dimensions also present a remarkable variability.

\subsection{Devices Metallization}

After printing, the devices were metallized. Unlike our previous paper on hydrogelcoated microdevices [32], the different metallic layers were not entirely applied by means of electroless deposition. Electrolytic deposition was used instead, following another previous paper published by our group [33]. By doing this, metallic layers characterized by more controllable properties and thickness were deposited. The first layer, due to the non-conductive nature of the SLA resin, was obligatorily applied by means of electroless deposition. Consequently, $400 \mathrm{~nm}$ of $\mathrm{Cu}$ were applied via immersion for $15 \mathrm{~min}$ in a formaldehyde-free copper electroless bath. Then, once the surface was conductive, the barrel-plating approach was employed to apply CoNiP and gold from electrolytic plating. This technology, which takes inspiration from the industrial practice used to coat small objects and is extensively described in our previous publication [33], allows electrolytic deposition without directly contacting each device. In turn, they are placed inside an electrified metallic basket and plated all together, exploiting the labile electrical connection between the devices and the walls of the basket (Figure 2a). A total of $5 \mu \mathrm{m}$ of CoNiP, whose ferromagnetic properties were exploited to make the devices actuable via magnetic fields, were deposited on the microrobots in $160 \mathrm{~min}$.

With regard to the barrel plating approach, we observed in our previous work [33] that the non-ideal contact between the devices and the basket requires the introduction of a correction coefficient (named $\psi$ ) in the standard well-known Faraday law Equation (1). The relationship describes the amount of material deposited with respect to the total charge used.

$$
m=\frac{M q}{Z F} \eta \psi
$$

$m$ is the total mass reduced, $M$ is the molar mass, $q$ is the total charge, $Z$ is the valence of the ions reduced, $F$ is the Faraday constant, $\eta$ is the cathodic efficiency of the reaction. $\psi$, as previously stated, is an apparent efficiency of the barrel process, which must be determined by comparing the amount of metal deposited in the barrel and the corresponding amount plated in analogous conditions on a standard planar surface with ideal electrical connection to the generator. $\eta$ and $\psi$ for CoNiP deposition were determined previously [33] and they resulted equal to 0.42 and 0.25 , respectively. In other words, only $10.5 \%$ of the current in the barrel is used to actually plate CoNiP on the devices. However, this apparent 
disadvantage of the barrel-plating approach is counterbalanced by the possibility to plate layers on small objects using electrolytic deposition and without leaving uncoated areas due to electrical contacting.
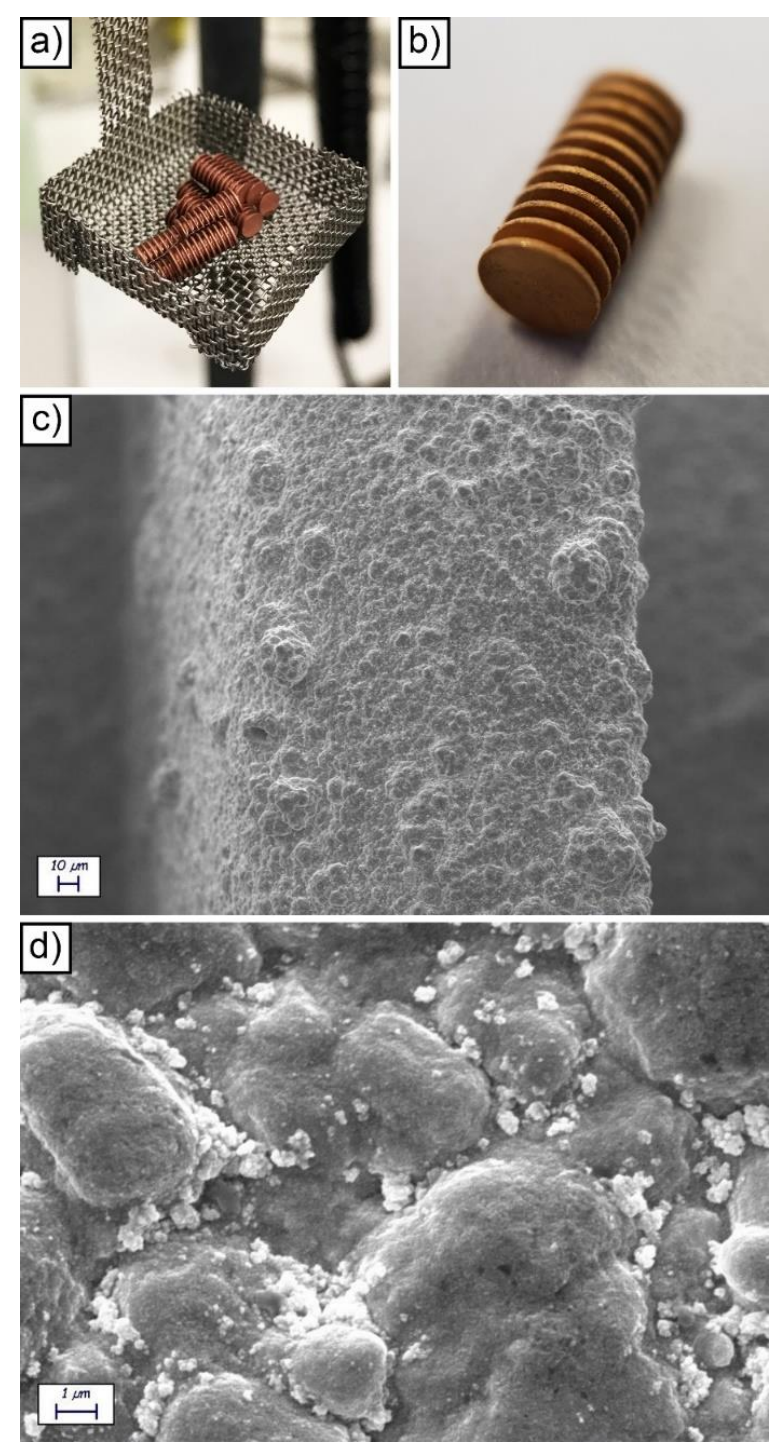

Figure 2. Visual appearance of the barrel setup used to metallize the devices (a); visual appearance of a Cu/CoNiP/Au coated device (b); SEM images of a metallized device observed at $1000 \mathrm{X}(\mathbf{c})$ and $20,000 \times(d)$.

At the end of the CoNiP plating step, the microdevices were coated with a 4- $\mu \mathrm{m}$ thick gold top layer to make the surface biocompatible and to avoid corrosion. Such a layer was again deposited using the barrel approach in a non-cyanide gold-plating bath. With respect to the galvanic displacement methodology previously employed to apply gold [22], electrolytic deposition allows to deposit thick layers (in the few $\mu \mathrm{m}$ range). Increasing the gold thickness renders the devices more resistant to corrosion, thus increasing the number of reuse cycles. Barrel gold deposition was carried out here for the first time and, for this reason, $\eta$ and $\psi$ were determined. $\eta$ of the deposition process was evaluated depositing gold on a planar copper substrate and the result was equal to 0.57 . $\psi$ was evaluated by weighing the devices before and after deposition in the barrel, considering the cathodic efficiency previously calculated and comparing the result with the mass deposited on the planar copper sample. The correction coefficient $\psi$ resulted equal to 0.28 . Gold was deposited for $220 \mathrm{~min}$ to obtain the thickness required. 
The final visual appearance of a gold-coated device is visible in Figure $2 b$. The surface appears uniformly covered with gold, as further certified by the SEM analysis (Figure 2c, d and Figure S5) and by the EDS characterization (Figure S6). As expected, the application of a metallic layer on the surface of the devices increased the roughness of the surface from $191 \pm 23 \mathrm{~nm}\left(\mathrm{R}_{\mathrm{a}}\right.$ for the surface of the uncoated resin) to $356 \pm 55 \mathrm{~nm}\left(\mathrm{R}_{\mathrm{a}}\right.$ for the surface $\mathrm{Cu} / \mathrm{CoNiP} / \mathrm{Au}$ coated devices). SEM analysis evidenced a nodular morphology on the surface of the devices, which was a result of the electrolytic deposition process employed for their metallization.

\subsection{Hydrogels Application}

At the end of the metallization process, microdevices were coated with alginate and the effect of the three different physical cross-linkers was examined. Considering the results obtained in different papers [37], the concentration of sodium alginate was fixed at $2 \% w / v$ in distilled water. Too high alginate concentrations lead to stiff hydrogels, which show higher rigidity but lower swelling abilities, thus compromising the drug loading capacity and its utilization inside the human body. Furthermore, the solutions with higher concentration are too viscous with a very long time of complete dissolution, and do not facilitate the deposition of the hydrogel on the device. Indeed, by increasing the alginate concentration, there are more polar groups (-COO-) interacting with divalent ions of the cross-linker agent, increasing the cross-linking density [38]. The crosslinking process keeps the chains closer to each other, increasing the mechanical properties of the devices but reducing their water and drug uptake. Lower concentrations produce, instead, a hydrogel with an excessive swelling degree, thus decreasing the adhesion to the substrate, increasing the volume and having inconsistent shapes with the magnetic actuation. Therefore, the final choice resulted from a balance between the swelling degree and the adhesion and consistency of the hydrogel over the scaffold.

In the present work, only the type of cross-linker was varied to evidence its effect on release properties. In general, alginate has the ability to form a 3D hydrogel network when in contact with divalent cations (such as $\mathrm{Ca}^{2+}, \mathrm{Fe}^{2+}, \mathrm{Mn}^{2+}, \mathrm{Ba}^{2+}, \mathrm{Sr}^{2+}$ ). Here, in analogy with Da Silva et al. [34], three different biocompatible cross-linker agents have been tested: $\mathrm{MnCl}_{2}, \mathrm{ZnCl}_{2}$ and $\mathrm{CaCl}_{2}$. The relative stoichiometry of the crosslinker was kept constant at a value of $1 \% w / v$, which has been reported in many works as optimal from the swelling, uniformity and stability point of view $[34,37,38]$. Indeed, hydrogels should remain attached over the scaffolds, without collapsing, with a thickness as uniform as possible, and they must permit a good uptake of water to guarantee a sufficient loading of drug molecules. Low cross-linker concentration means a lower diffusion gradient that leads to low rates and low degrees of crosslinking. This implies a very high swelling degree, excessive volume and poor homogeneity. On the contrary, excessive cross-linking can reduce the soft nature of the system needed for applications with living tissue. Lastly, higher concentrations of the crosslinking agent favor its diffusion among alginate chains and determine a faster crosslinking process.

Devices were suspended to a thin wire and sequentially immersed in the alginate solution and then in the crosslinking solution (Figure S7). To sustain the wire, a self-designed holder (Figure S8) was employed. In line with our previous work [32], the total thickness of the hydrogel layer could be increased by repeating the coating sequence. However, as evidenced in Figure $3 \mathrm{a}-\mathrm{d}$ for the $\mathrm{Ca}^{2+}$ reticulated alginate, the application of more than one layer progressively altered the shape of the devices. With one layer (Figure 3a) and two layers (Figure 3b), the shape of the devices remained relatively cylindrical. At three (Figure 3c) and four (Figure 3d) layers, the final shapes of the devices were strongly rounded by the surface tension of the non-reticulated hydrogel. The ellipsoidal shape resulting from more than two coating sequences could potentially interfere with device actuation, which was designed for cylindrical-shaped devices. For this reason, the number of hydrogel layers was limited to a maximum of two, ideally one. With this consideration, it is fundamental to load the highest possible amount of hydrogel to maximize the amount of loadable drug. 
The shape of the device has a strong influence on this aspect, as demonstrated by calculating the weight difference between uncoated and single layer coated devices. Hydrogel uncoated devices were characterized by a weight of $31.46 \pm 1.78 \mathrm{mg}$. Considering $\mathrm{Ca}^{2+}$ as a crosslinker, the final weight of the loaded hydrogel was $31.5 \pm 5.5 \mathrm{mg}$. This translated into a $100.13 \%$ increase of the weight after coating. If compared with the increase registered in our previous work [32] with porous microdevices $(80 \%)$, this value demonstrates that the honey dipper design is able to optimize hydrogel loading after a single coating step. Besides the device shape, the crosslinker employed also influenced the final loaded weight. Indeed, Figure $3 e$ depicts the weight increase recorded in the case of alginate applied on the microdevices and reticulated with $\mathrm{Ca}, \mathrm{Zn}$ and $\mathrm{Mn}$. A clear influence of the reticulating agent can be observed, with Mn allowing the lowest amount of loaded hydrogel $(14 \pm 0.5 \mathrm{mg})$.

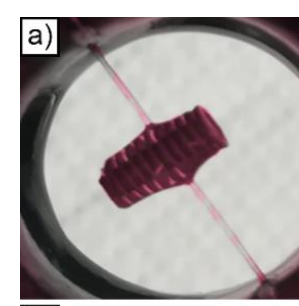

e)

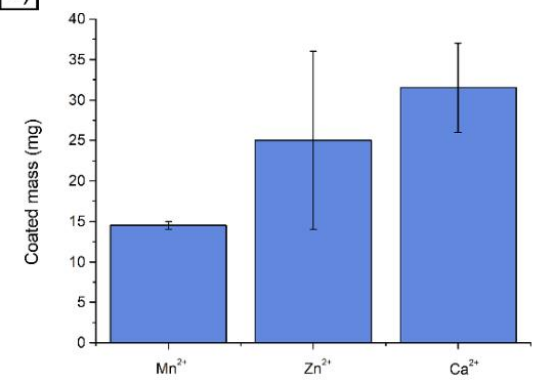

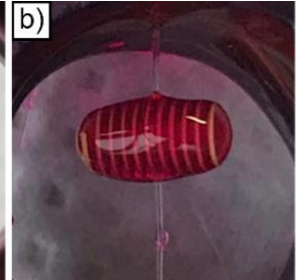

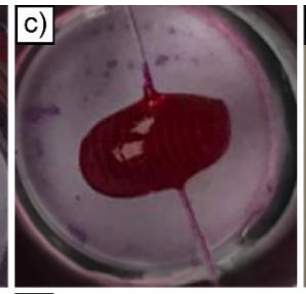

f)

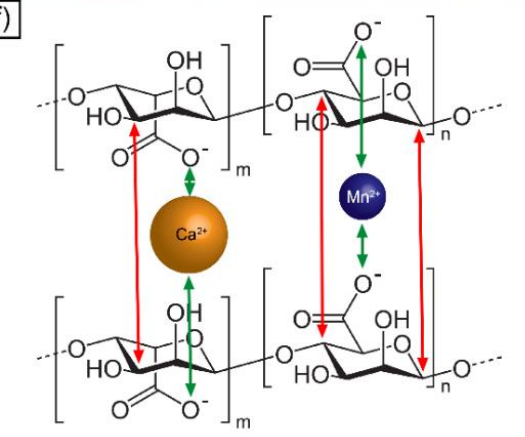

Figure 3. Visual appearance of devices coated with Ca-reticulated hydrogel after one (a), two (b), three (c) and four (d) coating cycles; amount of applied hydrogel after a single coating cycle as a function of the physical crosslinker employed (e); schematic representation of two alginate chain crosslinked with either Ca or Mn (f).

From the results obtained by Da Silva et al., it was possible to see that the hydrogel prepared with $\mathrm{Mn}^{2+}$ had a higher swelling degree if compared to those prepared with $\mathrm{Zn}^{2+}$ or $\mathrm{Ca}^{2+}$. As the three cross-linkers have the same valence $\left({ }^{2+}\right)$, the swelling variation can only be attributed to the different size of the ions- $-0.067,0.074$ and $0.100 \mathrm{~nm}$ for $\mathrm{Mn}, \mathrm{Zn}$ and $\mathrm{Ca}$, respectively. Ions with a larger radius have a higher interaction with the groups -COO- of sodium alginate, thus generating chains with higher entanglement (Figure 3f). The stronger interactions of the larger ions with the polymeric chain may be related to the polarizability and London dispersion forces. Larger molecules, atoms or ions usually exhibit higher London dispersion forces and are also more polarizable, thus increasing ion-dipole interactions with the polymer chain. These interactions minimize the elasticity of the polymeric chain, reducing its swelling degree. In addition, as is evident from Figure 3f, larger ions occupy a larger space in the empty interstices among the polymer chains (or pores), decreasing the virtual volume which could be occupied by water molecules. Therefore, due to their size, calcium ions are more efficient (in the same volume) in the polymeric chain entanglement, also providing more intermolecular interactions and limiting the expansion of the polymeric chain [34]. On the contrary, $\mathrm{Mn}^{2+}$ ions, due to the lowest ionic radius compared to the others, present a weak interaction with the -COO-groups of the alginate. This fact limits the effective crosslinking but improves the water absorption rate. This leads to less rigid hydrogels with polymer chains relatively free to move. 
From these considerations, it can be inferred that for the affinity of the ions with -COOgroups, the best crosslinkers, in order, are: $\mathrm{Ca}^{2+}>\mathrm{Zn}^{2+}>\mathrm{Mn}^{2+}$. From the experiments, it was also noticed that the mass of hydrogel loaded over the scaffold seems dependent on the type of crosslinker agent used. In particular, it also seems that the amount of gel loaded on the scaffold follows the same scale of affinity. As shown in Figure $3 \mathrm{e}, \mathrm{Ca}^{2+}$ cations, with the same procedure, allow to load more gel over the scaffold with respect to the two other ions, all without losing good homogeneity and good stability properties.

Another aspect to take into consideration is the effect of $\mathrm{pH}$ on the swelling behavior. In agreement with the results obtained by Da Silva et al. for the hydrogels crosslinked with $\mathrm{Mn}^{2+}$, it does not seem possible to measure the water absorption at different $\mathrm{pH}$ levels since the hydrogels dissolved during the first hours of the study. This result is interesting because it shows that the physical interactions generated in the synthesis of the hydrogels (using $\mathrm{Mn}^{2+}$ as crosslinking agent) can be easily broken. This thermodynamic instability was attributed to the competition between the $\mathrm{H}^{+}$ions of the swelling medium and the $\mathrm{Mn}^{2+}$ ions. In particular, the carboxylic groups responsible of the interactions with the divalent ions $\mathrm{Mn}^{2+}$ in the crosslinking process started to interact with the monovalent cationic ions of the medium $\left(\mathrm{Na}^{+}, \mathrm{H}^{+}\right)$, in the form of carboxylic acid or carboxylic acid salt $(-\mathrm{COOH},-\mathrm{COONa})$ groups, destroying the three-dimensional network. As to the effect of $\mathrm{pH}$ on the hydrogels crosslinked with $\mathrm{Zn}^{2+}$ or $\mathrm{Ca}^{2+}$, just a change in the swelling behavior, instead of a complete dissolution, was verified. This is due to the interactions of carboxylic groups with these two cations that are stronger and therefore only partially altered by $\mathrm{Na}^{+}$or $\mathrm{H}^{+}$action.

\subsection{Drug Release Performances}

Considering the different swelling behavior observed by Da Silva et al. [34] for the three different crosslinkers, an observable difference was also expected in the case of drug release performances. RhB release was investigated and the behavior of the three hydrogels was compared. Release studies were conducted at $37^{\circ} \mathrm{C}$ and $\mathrm{pH} 7.4$, with the results visible in Figure $4 \mathrm{a}$. The percentage of $\mathrm{RhB}$ released was defined as the ratio between the released amount in the aqueous media and the total amount loaded within the polymeric layer.

It appears evident that, contrary to what expected, the three materials followed a comparable trend, which led to an almost complete release of RhB after roughly two hours. This is in accordance with the fact that RhB presents a very small hydrodynamic radius and its release is not influenced by the different cross-linkers used. Information about the kinetics of the release can be extracted from the data visible in Figure $4 \mathrm{a}$ if the same are plotted against the time square root (Figure $4 \mathrm{~b}$ ). The initial linear part of the release curve is indicative of Fickian diffusion and the $y$-axis intercept value is an indication of burst release. The latter is relatively limited and took place when RhB-loaded devices were placed in the releasing medium. After the initial fast release, the RhB loaded within the three alginate hydrogels showed a marked linear trend only in the first $2 \mathrm{~h}$. This corresponds to a pure Fickian diffusion and is only driven by the concentration gradient. Afterwards, the trend reached a plateau. Indeed, when the main release was completed, the residual drug entrapped within the hydrogel network was slowly released.

The data obtained were used to estimate RhB diffusion coefficients. Operatively, the release mechanism could be considered as a pure Fickian diffusion, the concentration being driven through alginate. Under this assumption, the drug diffusion kinetic can be described as a one-dimensional model of the second Fick law where the device geometry is a cylinder and the material flux mainly takes place at the PBS/hydrogel surface. Equation (2) showed these considerations, indicating $r$ as the characteristic radius for the mass transport phenomenon. The following mass balance equations are written considering the variation of the mean drug concentration within the hydrogel $\left(C_{G}\right)$ related to the volume of solution $\left(V_{S}\right)$, the mean drug concentration in the outer solution $\left(C_{S}\right)$, the total volume $\left(V_{G}\right)$, the drug present inside the matrix $\left(m_{G}\right)$ and the exchange interfacial surface $\left(S_{\text {exc }}\right)$, which represents the boundary surface between the device and the surrounding solution (which, simplifying, 
can here be considered as being only the side surface). According to these expressions, the boundary conditions are defined describing the profile symmetry at the center of the polymeric cylinder with respect to the radial axis of the cylinder-Equation (6) and the equivalence between the material diffusive fluxes at the PBS/hydrogel surfaceEquation (7).

$$
\begin{gathered}
\frac{\partial C_{G}}{\partial t}=D \cdot \frac{1}{r^{2}} \cdot \frac{\partial}{\partial r} \cdot\left(r^{2} \cdot \frac{\partial C_{G}}{\partial r}\right) \\
V_{S} \frac{\partial C_{G}}{\partial t}=k_{C} \cdot S_{e x c} \cdot\left(C_{G}-C_{S}\right) \\
C_{S}(t=0)=0 \\
C_{G}(t=0)=C_{G, 0}=\frac{m_{G, 0}}{V_{G}} \\
\left.\frac{\partial C_{G}}{\partial r}\right|_{r=0}=0 \\
-\left.D \cdot \frac{\partial C_{G}}{\partial r}\right|_{r=R}=k_{C} \cdot\left(C_{G}-C_{S}\right)
\end{gathered}
$$

a)

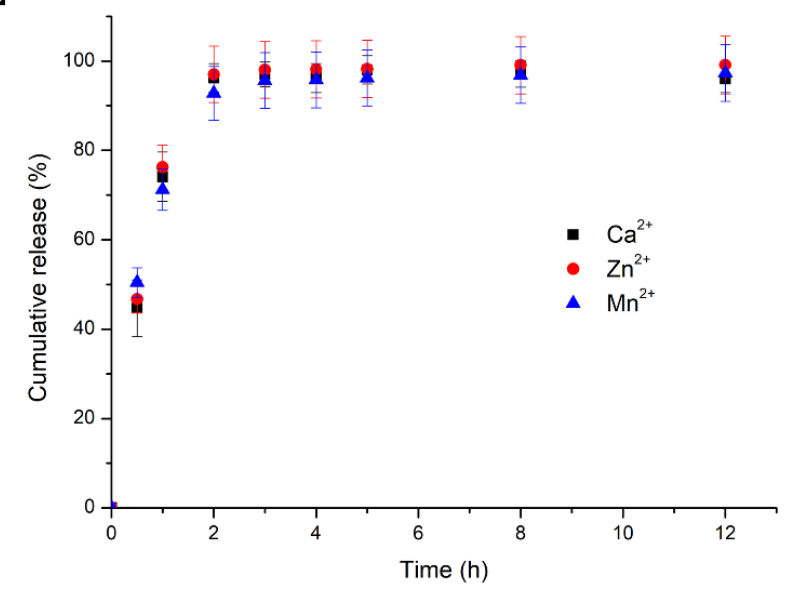

b)

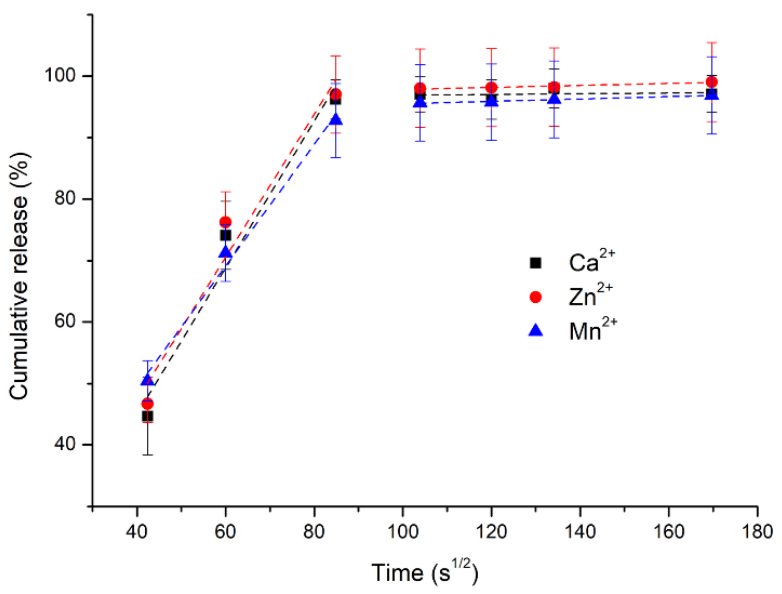

Figure 4. RhB release results obtained at $37^{\circ} \mathrm{C}$ and $\mathrm{pH} 7.4$ with different physical crosslinkers (a); $\mathrm{RhB}$ release results obtained at $37^{\circ} \mathrm{C}$ and $\mathrm{pH} 7.4$ with different physical crosslinkers plotted against time square root $(\mathbf{b})$. 
This mathematical model allowed to estimate the diffusion coefficient $(D)$ of RhB. The Sherwood number obtained by means of penetration theory allowed the computation of the mass transfer coefficient $k_{C}$-Equation (8).

$$
S h=1=\frac{k_{C} \cdot 2 r}{D}
$$

Table 1 resumes the results obtained.

Table 1. Diffusion coefficient of RhB in alginate hydrogels reticulated with different cross-linkers.

\begin{tabular}{cc}
\hline Title $\mathbf{1}$ & Diffusivity $\left[\mathrm{cm}^{2} / \mathbf{s}\right]$ \\
\hline Ca reticulated alginate & $2.5610^{-4}$ \\
Zn reticulated alginate & $2.710^{-4}$ \\
Mn reticulated alginate & $2.5710^{-4}$ \\
\hline
\end{tabular}

As expected from the data reported in Figure 4, the three diffusion coefficients are comparable. This result confirms the limited effect induced by the difference in ionic size of the three crosslinkers on the Rhb release.

Apparently, by considering the results obtained, the most advantageous crosslinking agent for alginate is calcium. Indeed, devices can be loaded with a higher amount of hydrogel, in front of a substantially similar release rate. However, the particular $\mathrm{pH}$ dependent behavior of alginate reticulated with $\mathrm{Mn}^{2+}$ can be potentially exploited for $\mathrm{pH}$-triggered drug release. The idea, hereby suggested for the first time and qualitatively demonstrated, is that a device coated with $\mathrm{Mn}^{2+}$ reticulated alginate can almost instantaneously release all its drug load when it reaches an environment characterized by an acidic $\mathrm{pH}$ (e.g., the stomach). Indeed, acid degradable polymers present interesting properties for drug delivery $[39,40]$. Diffusion-driven discharge of a drug from a hydrogel is governed by n-order release kinetics [41], which are in the vast majority of the cases desirable to achieve sustained release. Degradable materials, however, are highly attractive when steep release gradients must be provided (with almost instantaneous release of the drug) [42]. This is the case for drugs that require high instantaneous concentrations to carry out their therapeutic action. Considering a possible application for $\mathrm{pH}$-triggered drug release, the remaining experimentation presented in the paper was carried out on Mn-containing alginate devices.

\subsection{Magnetic Actuation}

Prior to functionality evaluation, the magnetic maneuverability of the devices was evaluated. Samples coated with Mn-reticulated alginate were placed inside a waterfilled basin (to avoid hydrogel desiccation) and actuated, applying a rotating magnetic field. The principle exploited to move the microrobots is the continuous application of torque on the magnetic material present in the devices to generate a rolling motion. This actuation route, employed also in our previous works [22,33], requires the presence of a permanently magnetizable material. Consequently, an alloy like CoNiP (hard magnetic at the composition employed) was selected. As visible in Figure S9, which depicts the VSM performed on a single device, CoNiP presented a remanence in the order of $1124 \mathrm{Oe}$ (along the $0^{\circ}$ direction) and $936 \mathrm{Oe}$ (along the $90^{\circ}$ direction). In the convention employed for the VSM test, $0^{\circ}$ refers to the direction parallel to the symmetry axis of the device, while $90^{\circ}$ is the direction perpendicular to the same axis. These high remanence values are a consequence of the composition of the alloy: 3.08\% wt. P, 84.75\% wt. Co and $12.24 \%$ wt. Ni. Electrodeposited CoNiP layers presenting this composition are characterized by a typical hexagonal close-packed (hcp) structure with a marked preferential orientation along the (002), which justify the high level of coercivity and remanence achievable [43].

During rolling actuation, the permanent magnetization vector $M$ introduced in the device by placing it in contact with a permanent NdFeB magnet continuously aligns itself (Figure $5 a$ ) with the external rotating field $B$. In this way, the device rotates around 
its symmetry axis (marked with a dashed black line in Figure 5a). The contact with a solid surface generates a net forward motion on the device. If the orientation of the axis with $B$ rotates is varied, the direction of the motion can be adjusted and the device can be steered (Figure 5a). Obviously, in analogy with a wheel, there is a direct dependency between the rotation frequency of the magnetic field and the forward speed of the device. Under the assumption of an ideal contact between the device and the substrate, the speed of the first $(v)$ correlates to the frequency $(\theta)$ according to Equation (9).

$$
v=2 \pi r \theta
$$

$r$ corresponds to the radius of the device (comprising the rigid part of the device and the hydrogel layer deposited on top). Consequently, the speed/frequency relationship should be linear at any frequency. As demonstrated in our previous paper [32], this is never true for hydrogel-coated devices due to the presence of the hydrogel itself. Figure $5 b$ reports the results obtained by performing a linear actuation on a device at increasing frequency. The data clearly evidenced that the uncoated device almost perfectly followed a linear behavior (evidenced by the fitting performed). The hydrogel-coated device, on the contrary, deviated from the linearity at frequencies higher than $2 \mathrm{~Hz}$. The linear fitting was, in this case, limited to the 0 to $2 \mathrm{~Hz}$ range.
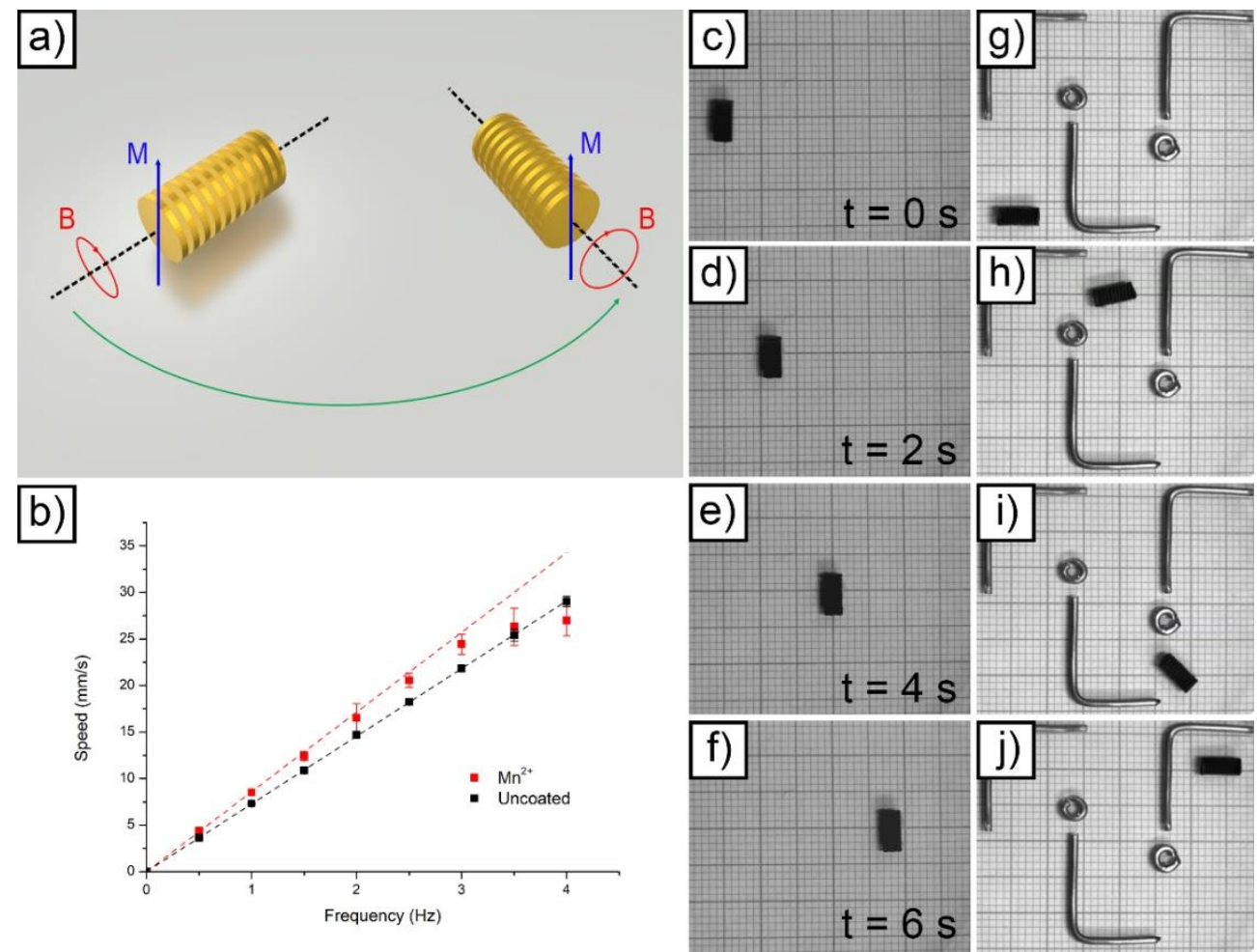

Figure 5. Schematic representation of the magnetic actuation principle employed for microrobots actuation (a); speed vs. frequency relationship for uncoated and Mn alginate-coated microdevices (b); linear actuation of a single Mn alginate-coated device (from (c-f)); remote guidance of a Mn alginatecoated device to avoid metallic obstacles (from $(\mathbf{g}-\mathbf{j})$ ).

Considering Equation (9), it appears evident that the slope of the fitted curves visible in Figure $5 \mathrm{~b}$ corresponds to $2 \pi r$. Consequently, the radius of the devices (resulting from the sum between the radius of rigid platform and the radius of the hydrogel layer applied on top) was easily extrapolated from the slope of the fitted curves. The result was equal to $1.161 \mathrm{~mm}$ for the uncoated device and to $1.364 \mathrm{~mm}$ for the coated device. The value obtained for the uncoated microrobot is in line with the theoretical one reported in Table S1 $(1.1975 \mathrm{~mm})$. The value obtained in the case of the coated device suggests that the thickness 
of the Mn-reticulated hydrogel layer was equal to $203 \mu \mathrm{m}$. This value does not correspond to the absolute value of thickness for the hydrogel coating. Inside the plates, for example, this value was realistically different due to hydrogel accumulation. The value obtained represents a mean hydrogel thickness in correspondence with the external radius of the devices (on the parallel plates).

Figure $5 \mathrm{c}-\mathrm{f}$ and Supporting Video S1 depict the linear actuation performed to obtain the speed data at $0.5 \mathrm{~Hz}$ in Figure 5b. The linear correlation between elapsed time and covered length was evidenced by showing four different frames acquired at increasing times. Figure $5 \mathrm{~g}-\mathrm{j}$ and Supporting Video S2 report the result obtained by guiding a $\mathrm{Mn}$ reticulated alginate-coated device to avoid metallic obstacles placed inside the water-filled basin. The device evidenced an excellent maneuverability at $0.5 \mathrm{~Hz}$ actuation frequency.

\subsection{Targeted $p H$ Dependent Release}

To experimentally validate the basic concept of targeted $\mathrm{pH}$-dependent delivery from a qualitative point of view, two distinct tests were carried out. Initially, the capacity of targeting drug delivery of the devices was tested. A Mn-reticulated alginate-coated device, suitably loaded with RhB, was placed inside a basin and actuated. UV light was exploited to evidence, thanks to $\mathrm{RhB}$ fluorescence at $345 \mathrm{~nm}$, the presence of the drug on the walls of the basin. The result obtained is presented in Figure 6a-f and in Supporting Video S3. At the beginning of the test, the device moved towards the center of the basin along a curved trajectory. Once it reached the center of the basin, the device was left there for a few seconds to allow release. Then, the device was moved, always along a curved trajectory, toward the lower corner of the basin. Besides the tracks left in the water, UV light evidenced the presence of $\mathrm{RhB}$ adsorbed on the bottom of the basin in correspondence with the point where the device stayed for a few seconds. This qualitative result suggests the idea that these devices could be potentially guided in vivo inside the gastrointestinal tract, left in contact with the inner parts of the organs to locally favor the absorption of a drug of choice.
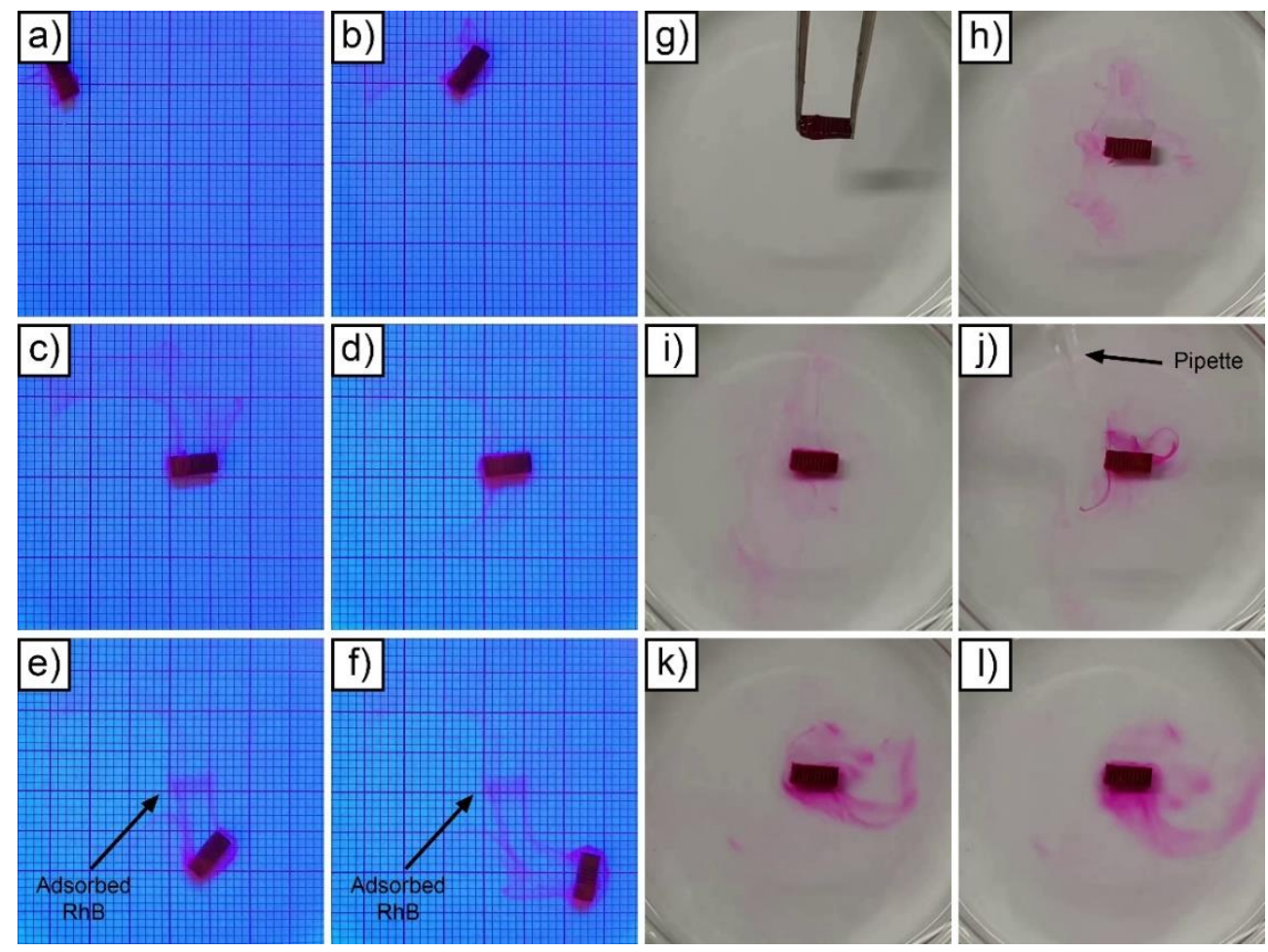

Figure 6. Targeted drug delivery from a device (from (a-f)); Mn-reticulated hydrogel dissolution test at $\mathrm{pH} 3$ (from $(\mathbf{g}-\mathbf{l}))$. 
In the second test, visualized in Figure $6 \mathrm{~g}-1$ and in Supporting Video S4, a device analogous to the one used for the previous test was immersed in water. As expected, the microrobot immediately started releasing RhB according to a Fickian diffusion mechanism. In these conditions, nearly total release of the drug potentially required almost two hours (as visible in Figure $4 \mathrm{a}$ ). To exponentially increase the release rate, thus allowing immediate drug release, the $\mathrm{pH}$ of the water-filled basin was artificially lowered by adding $\mathrm{HCl}$. Specifically, the stomach environment was simulated, reaching $\mathrm{pH} 3$ [44]. To obtain this condition, a suitable quantity of $\mathrm{HCl}$ was added to the basin and rapidly mixed with a pipette. Under these conditions, the device started to quickly release all the RhB contained inside the alginate layer, which rapidly disintegrated in a few-minute timeframe.

Thanks to the low $\mathrm{pH}$, the device expelled its RhB load almost immediately, with a drug release not governed by diffusion in the hydrogel. Furthermore, hydrogel removal by $\mathrm{HCl}$ allowed direct reuse of the device.

\subsection{Possible Toxicity of Mn Reticulated Alginate}

Possible dose-dependent Mn toxicity is a matter of major importance when evaluating the potential use of Mn-reticulated alginate for drug delivery applications. In fact, when the acidity mechanically degrades the alginate layer, all the Mn contained is immediately released in the environment. $\mathrm{Mn}$ is an element required in small quantities by the human body [45], but its excess may potentially result in a condition known as manganism. Mn is normally obtained through dietary intake and is readily absorbed by the gastrointestinal apparatus. $\mathrm{O}^{\prime} \mathrm{Neal}$ et al. evidenced that the average daily intake for many Western diets is between 2.3 and $8.8 \mathrm{mg}$ [45]. Conversely, the WHO standard for drinking water is $400 \mu \mathrm{g} / \mathrm{L}$ of Mn [45]. These values, which represent safe Mn consumption from food and drinking water, can be compared with the mean amount of Mn released by a device when the alginate layers dissolve. Each device is coated with $14 \pm 0.5 \mathrm{mg}$ of Mn-reticulated hydrogel (data from Figure 3). According to the supplier (Sigma Aldrich), the mean molecular weight of the alginate employed ( $\left.M W_{\text {hydrogel }}\right)$ was between 120 and $190 \mathrm{kDa}(120 \mathrm{kDa}$ was considered). The total amount of Mn within every device can be obtained considering the number of monomeric units present in $14 \mathrm{mg}$ of the hydrogel layer. The percentage of polymer with respect to water in these hydrogels is approximately $20 \% \mathrm{wt}$., so the number of monomers is approximately $8 \times 10^{18}$. Taking into consideration the ratio between one atom of $\mathrm{Mn}$ every two monomeric units of alginate, we can obtain the Mn amount, which is around $350 \mu \mathrm{g}$. This value is lower than the tolerable amount recommended by the WHO for a liter of drinking water $(400 \mu \mathrm{g})$. In front of these realistic considerations, the modest amount of Mn released from a single device cannot be considered harmful for the human body.

\section{Discussion}

The experiments carried out in the present work expanded the possibilities for targeted drug release from hydrogel layers loaded on magnetically moveable rigid platforms. Initially, the hydrogel loading capability of the devices was optimized by introducing a honey dipper design. This approach resulted in a $20 \%$ load increase with respect to our previous design. Microdevices were successfully 3D printed via stereolithography and wet metallized with a sequence of functional layers. The use of barrel plating allowed to deposit thick metallic layers of $\mathrm{CoNiP}$ and $\mathrm{Au}$ without direct clamping of the single device (which would have resulted in uncoated areas). After plating, the devices were successfully coated with a controllable amount of alginate hydrogel. The type of crosslinker employed for reticulation was found to have a remarkable influence on the amount of hydrogel deposited on a single device, with Mn allowing the lowest amount of loaded hydrogel. On the contrary, the three different reticulating agents evidenced a limited effect on drug release performances. Mn alginate-coated devices were actuated applying external rotating magnetic fields, evidencing a good control over speed and position. Even though less advantageous in terms of loadable hydrogel amount, Mn-reticulated alginate was characterized by an interesting property: the layer dissolved at low $\mathrm{pH}$, immediately 
releasing all its drug load. The possible applicability of this property, which can potentially be exploited to trigger an instantaneous release by mean of $\mathrm{pH}$ variation, was successfully demonstrated. A single device bearing Mn-reticulated hydrogel was subjected to a controlled $\mathrm{pH}$ variation simulating the gastric environment. The result obtained qualitatively evidenced the possibility of $\mathrm{pH}$-triggering an instantaneous release, characterized by steep release rates and high instantaneous drug bioavailability. These features may be useful for specific types of drugs that present high active concentrations. The results obtained suggest the idea that hydrogels reticulated with ions alternative to the classically employed Ca can be used to implement a $\mathrm{pH}$-dependent release. They constitute a significant base for further experiments, which are currently under evaluation. Specifically, Mn-reticulated layers could be coupled with Ca-reticulated ones to tune the release. In addition, alternative small-sized biocompatible ions (or mixtures of different ions) could be evaluated to tune the $\mathrm{pH}$ sensitivity of the hydrogel layer.

Supplementary Materials: The following are available online at https:/ /www.mdpi.com/article/10 $.3390 /$ technologies $9020043 / \mathrm{s} 1$, Table S1: Theoretical and experimental dimensions of the microdevices, Figure S1: Honey dipper, Figure S2: dimensions of the devices, Figure S3: SEM image of an as printed device (10,000 X), Figure S4: Section of a metallized device (optical microscope), Figure S5: SEM image of a metallized device (10,000 X), Figure S6: EDS analysis of the surface depicted in Figure S5, Figure S7: Hydrogel loading procedure, Figure S8: Sample holder, Figure S9: VSM of an uncoated device, Supplementary Video S1, Supplementary Video S2, Supplementary Video S3, Supplementary Video S4.

Author Contributions: Conceptualization: R.B. and F.R.; methodology: R.B. and F.R.; validation: R.B., F.R., A.R. and F.P.; investigation: R.B., F.P., A.R., R.P. and A.N.; resources: M.L.; writing—original draft preparation: R.B. and F.R.; writing — review and editing: R.B., F.R. and A.N.; supervision: R.B. and F.R. All authors have read and agreed to the published version of the manuscript.

Funding: This research received no external funding.

Institutional Review Board Statement: Not applicable.

Informed Consent Statement: Not applicable.

Data Availability Statement: The data that support the findings of this study are available from the corresponding author, R. Bernasconi, upon reasonable request.

Acknowledgments: The authors wish to acknowledge the support given by Salvador Panè (ETH Zurich), who provided access to the Octomag magnetic manipulation system and to the VSM setup employed in the paper.

Conflicts of Interest: The authors declare no conflict of interest.

\section{References}

1. Brater, D.C.; Daly, W.J. Clinical pharmacology in the Middle Ages: Principles that presage the 21st century. Clin. Pharmacol. Ther. 2000, 67, 447-450. [CrossRef]

2. Abu-Thabit, N.Y.; Makhlouf, A.S.H. Historical Development of Drug Delivery Systems: From Conventional Macroscale to Controlled, Targeted, and Responsive Nanoscale Systems; Elsevier: Amsterdam, The Netherlands, 2018; ISBN 9780081019979.

3. Wang, B.; Hu, L.; Siahaan, T.J. Drug Delivery: Principles and Applications; John Wiley \& Sons: Hoboken, NJ, USA, 2016; ISBN 1118833236.

4. Sekyere, J.O.; Asante, J. Emerging mechanisms of antimicrobial resistance in bacteria and fungi: Advances in the era of genomics. Future Microbiol. 2018, 13, 241-262. [CrossRef]

5. Vasan, N.; Baselga, J.; Hyman, D.M. A view on drug resistance in cancer. Nature 2019, 575, 299-309. [CrossRef] [PubMed]

6. Holowka, E.P.; Bhatia, S.K. Smart Drug Delivery Systems. In Drug Delivery; Springer: Berlin/Heidelberg, Germany, 2014; pp. 265-316.

7. Alvarez-Lorenzo, C.; Concheiro, A. Smart drug delivery systems: From fundamentals to the clinic. Chem. Commun. 2014, 50, 7743-7765. [CrossRef] [PubMed]

8. Lee, Y.; Thompson, D.H. Stimuli-responsive liposomes for drug delivery. Wiley Interdiscip. Rev. Nanomed. Nanobiotechnol. 2017, 9, e1450. [CrossRef] [PubMed]

9. Pattni, B.S.; Chupin, V.V.; Torchilin, V.P. New developments in liposomal drug delivery. Chem. Rev. 2015, 115, 10938-10966. [CrossRef] [PubMed] 
10. Zelikin, A.N. Drug releasing polymer thin films: New era of surface-mediated drug delivery. ACS Nano 2010, 4, $2494-2509$. [CrossRef] [PubMed]

11. Hu, Q.; Li, H.; Wang, L.; Gu, H.; Fan, C. DNA nanotechnology-enabled drug delivery systems. Chem. Rev. 2018, 119, 6459-6506. [CrossRef] [PubMed]

12. Chauhan, A.S. Dendrimers for drug delivery. Molecules 2018, 23, 938. [CrossRef]

13. Zhou, Q.; Zhang, L.; Yang, T.; Wu, H. Stimuli-responsive polymeric micelles for drug delivery and cancer therapy. Int. J. Nanomed. 2018, 13, 2921. [CrossRef]

14. Manzano, M.; Vallet-Regí, M. Mesoporous silica nanoparticles for drug delivery. Adv. Funct. Mater. 2020, 30, 1902634. [CrossRef]

15. Vangijzegem, T.; Stanicki, D.; Laurent, S. Magnetic iron oxide nanoparticles for drug delivery: Applications and characteristics. Expert Opin. Drug Deliv. 2019, 16, 69-78. [CrossRef]

16. Ghitman, J.; Biru, E.I.; Stan, R.; Iovu, H. Review of hybrid PLGA nanoparticles: Future of smart drug delivery and theranostics medicine. Mater. Des. 2020, 193, 108805. [CrossRef]

17. Nelson, B.J.; Kaliakatsos, I.K.; Abbott, J.J. Microrobots for Minimally Invasive Medicine. Annu. Rev. Biomed. Eng. 2010, $12,55-85$. [CrossRef]

18. Chen, X.-Z.; Hoop, M.; Mushtaq, F.; Siringil, E.; Hu, C.; Nelson, B.J.; Pané, S. Recent developments in magnetically driven micro-and nanorobots. Appl. Mater. Today 2017, 9, 37-48. [CrossRef]

19. Jang, D.; Jeong, J.; Song, H.; Chung, S.K. Targeted drug delivery technology using untethered microrobots: A review. J. Micromech Microeng. 2019, 29, 53002. [CrossRef]

20. Erkoc, P.; Yasa, I.C.; Ceylan, H.; Yasa, O.; Alapan, Y.; Sitti, M. Mobile Microrobots for Active Therapeutic Delivery. Adv. Ther. 2019, 2, 1800064. [CrossRef]

21. Xu, T.; Yu, J.; Yan, X.; Choi, H.; Zhang, L. Magnetic actuation based motion control for microrobots: An overview. Micromachines 2015, 6, 1346-1364. [CrossRef]

22. Bernasconi, R.; Cuneo, F.; Carrara, E.; Chatzipirpiridis, G.; Hoop, M.; Chen, X.; Nelson, B.J.; Pané, S.; Credi, C.; Levi, M.; et al. Hard-magnetic cell microscaffolds from electroless coated 3D printed architectures. Mater. Horiz. 2018, 5, 699-707. [CrossRef]

23. Bernasconi, R.; Favara, N.; Fouladvari, N.; Invernizzi, M.; Levi, M.; Vidal, S.P.; Magagnin, L. Nanostructured Polypyrrole Layers Implementation on Magnetically Navigable 3D Printed Microdevices for Targeted Gastrointestinal Drug Delivery. Multifunct. Mater. 2020, 3, 045003. [CrossRef]

24. Jeon, S.; Kim, S.; Ha, S.; Lee, S.; Kim, E.; Kim, S.Y.; Park, S.H.; Jeon, J.H.; Kim, S.W.; Moon, C. Magnetically actuated microrobots as a platform for stem cell transplantation. Sci. Robot. 2019, 4, eaav4317. [CrossRef]

25. Cabanach, P.; Pena-Francesch, A.; Sheehan, D.; Bozuyuk, U.; Yasa, O.; Borros, S.; Sitti, M. Zwitterionic 3D-Printed NonImmunogenic Stealth Microrobots. Adv. Mater. 2020, 32, 2003013. [CrossRef]

26. Li, J.; Pumera, M. 3D printing of functional microrobots. Chem. Soc. Rev. 2021, 50, 2794-2838. [CrossRef]

27. Lee, H.; Kim, D.; Kwon, S.; Park, S. Magnetically Actuated Drug Delivery Helical Microrobot with Magnetic Nanoparticle Retrieval Ability. ACS Appl. Mater. Interfaces 2021, 13, 19633-19647. [CrossRef]

28. Park, J.; Kim, J.; Pané, S.; Nelson, B.J.; Choi, H. Acoustically Mediated Controlled Drug Release and Targeted Therapy with Degradable 3D Porous Magnetic Microrobots. Adv. Healthc. Mater. 2020, 10, 2001096. [CrossRef]

29. Lee, S.; Kim, J.; Kim, J.; Hoshiar, A.K.; Park, J.; Lee, S.; Kim, J.; Pané, S.; Nelson, B.J.; Choi, H. A Needle-Type Microrobot for Targeted Drug Delivery by Affixing to a Microtissue. Adv. Healthc. Mater. 2020, 9, 1901697. [CrossRef] [PubMed]

30. Narayanaswamy, R.; Torchilin, V.P. Hydrogels and their applications in targeted drug delivery. Molecules 2019, 24, 603. [CrossRef] [PubMed]

31. Sood, N.; Bhardwaj, A.; Mehta, S.; Mehta, A. Stimuli-responsive hydrogels in drug delivery and tissue engineering. Drug Deliv. 2016, 23, 748-770. [CrossRef] [PubMed]

32. Bernasconi, R.; Mauri, E.; Rossetti, A.; Rimondo, S.; Suriano, R.; Levi, M.; Sacchetti, A.; Pané, S.; Magagnin, L.; Rossi, F. 3D integration of $\mathrm{pH}$-cleavable drug-hydrogel conjugates on magnetically driven smart microtransporters. Mater. Des. 2021, 197, 109212. [CrossRef]

33. Bernasconi, R.; Carrara, E.; Hoop, M.; Mushtaq, F.; Chen, X.; Nelson, B.J.; Pané, S.; Credi, C.; Levi, M.; Magagnin, L. Magnetically navigable 3D printed multifunctional microdevices for environmental applications. Addit. Manuf. 2019, 28, 127-135. [CrossRef]

34. Da Silva Fernandes, R.; Tanaka, F.N.; de Moura, M.R.; Aouada, F.A. Development of alginate/starch-based hydrogels crosslinked with different ions: Hydrophilic, kinetic and spectroscopic properties. Mater. Today Commun. 2019, 21, 100636. [CrossRef]

35. Rizwan, M.; Yahya, R.; Hassan, A.; Yar, M.; Azzahari, A.D.; Selvanathan, V.; Sonsudin, F.; Abouloula, C.N. pH sensitive hydrogels in drug delivery: Brief history, properties, swelling, and release mechanism, material selection and applications. Polymers 2017, 9, 137. [CrossRef] [PubMed]

36. Kummer, M.P.; Abbott, J.J.; Kratochvil, B.E.; Borer, R.; Sengul, A.; Nelson, B.J. Octomag: An electromagnetic system for 5-DOF wireless micromanipulation. IEEE Trans. Robot. 2010, 26, 1006-1017. [CrossRef]

37. Dodero, A.; Pianella, L.; Vicini, S.; Alloisio, M.; Ottonelli, M.; Castellano, M. Alginate-based hydrogels prepared via ionic gelation: An experimental design approach to predict the crosslinking degree. Eur. Polym. J. 2019, 118, 586-594. [CrossRef]

38. Blandino, A.; Macias, M.; Cantero, D. Formation of calcium alginate gel capsules: Influence of sodium alginate and $\mathrm{CaCl}_{2}$ concentration on gelation kinetics. J. Biosci. Bioeng. 1999, 88, 686-689. [CrossRef] 
39. Binauld, S.; Stenzel, M.H. Acid-degradable polymers for drug delivery: A decade of innovation. Chem. Commun. 2013, 49, 2082-2102. [CrossRef] [PubMed]

40. Uhrich, K.E.; Abdelhamid, D. Biodegradable and Bioerodible Polymers for Medical Applications; Elsevier: Amsterdam, The Netherlands, 2016; ISBN 9781782421139.

41. Bruschi, M.L. Mathematical Models of Drug Release; Woodhead Publishing: Sawston, UK, 2015; ISBN 9780081000922.

42. Lao, L.L.; Peppas, N.A.; Boey, F.Y.C.; Venkatraman, S.S. Modeling of drug release from bulk-degrading polymers. Int. J. Pharm. 2011, 418, 28-41. [CrossRef]

43. Park, D.Y.; Myung, N.V.; Schwartz, M.; Nobe, K. Nanostructured magnetic CoNiP electrodeposits: Structure-property relationships. Electrochim. Acta 2002, 47, 2893-2900. [CrossRef]

44. Russell, T.L.; Berardi, R.R.; Barnett, J.L.; Dermentzoglou, L.C.; Jarvenpaa, K.M.; Schmaltz, S.P.; Dressman, J.B. Upper Gastrointestinal pH in Seventy-Nine Healthy, Elderly, North American Men and Women. Pharm. Res. 1993, 10, 187-196. [CrossRef]

45. O'Neal, S.L.; Zheng, W. Manganese Toxicity Upon Overexposure: A Decade in Review. Curr. Environ. Health Rep. 2015, 2, 315-328. [CrossRef] 